\title{
Prospects for Antineutrino Running at MiniBooNE
}

\author{
M.O. Wascko ${ }^{\text {a }}$ \\ ${ }^{a}$ Department of Physics and Astronomy, \\ Louisiana State University, Baton Rouge, LA 70803
}

We outline a program of antineutrino cross-section measurements necessary for the next generation of neutrino oscillation experiments, that can be performed with one year of data at MiniBooNE. We describe three independent methods of constraining wrong-sign (neutrino) backgrounds in an antineutrino beam, and their application to the MiniBooNE antineutrino cross section measurements.

\section{Introduction}

The search for $\mathrm{CP}$ violation in the neutrino sector requires both $\nu_{\mu} \rightarrow \nu_{e}$ and $\bar{\nu}_{\mu} \rightarrow \bar{\nu}_{e}$ oscillations measuremetns by future off-axis experiments. The signature for $\mathrm{CP}$ violation is an asymmetry in these oscillation probabilities, but this can only be confirmed if the precision of the $\nu$ and $\bar{\nu}$ cross sections are smaller than the observed asymmetry. There are few $\nu$ cross section data published [1] to date, but even fewer measurements of low energy $\bar{\nu}$ cross sections. We will need more and better data if we hope to find $\mathrm{CP}$ violation.

Table 1 lists the expected antineutrino event statistics for one year of $\bar{\nu}$ running $\left(2 \times 10^{20} \mathrm{POT}\right)$ with MiniBooNE [234]. Rates are listed for both right-sign (antineutrino,RS) and wrong-sign (neutrino,WS) interactions. Note that wrongsign comprise $30 \%$ of the total events. To constrain the wrong-sign backgrounds, MiniBooNE has developed new analysis techniques. We describe three methods below, and also describe their application to $\bar{\nu}$ cross section measurements at MiniBooNE.

\section{Constraining Wrong Sign Events}

For charged current (CC) interactions, neutrino events are typically distinguished from antineutrino events by identifying the charge of the outgoing muon. MiniBooNE, which has no magnetic field, has developed several novel techniques for measuring wrong-sign backgrounds in
Table 1

Event rates expected in MiniBooNE $\bar{\nu}$ running with $2 \times 10^{20}$ POT assuming a $550 \mathrm{~cm}$ fiducial volume, before cuts. Listed are the expected rightsign (RS) and wrong-sign (WS) events for each reaction channel. These event estimates do not include the effects of final state interactions in carbon nor the effects from event reconstruction.

\begin{tabular}{|c|c|c|}
\hline Reaction & $\bar{\nu}_{\mu}(\mathrm{RS})$ & $\nu_{\mu}(\mathrm{WS})$ \\
\hline $\mathrm{CC} \mathrm{QE}$ & 32,476 & 11,234 \\
\hline $\mathrm{NC}$ elastic & 13,329 & 4,653 \\
\hline $\mathrm{CC}$ resonant $1 \pi^{-}$ & 7,413 & 0 \\
\hline $\mathrm{CC}$ resonant $1 \pi^{+}$ & 0 & 6,998 \\
\hline CC resonant $1 \pi^{0}$ & 2,329 & 1,380 \\
\hline $\mathrm{NC}$ resonant $1 \pi^{0}$ & 3,781 & 1,758 \\
\hline $\mathrm{NC}$ resonant $1 \pi^{+}$ & 1,414 & 654 \\
\hline $\mathrm{NC}$ resonant $1 \pi^{-}$ & 1,012 & 520 \\
\hline $\mathrm{NC}$ coherent $1 \pi^{0}$ & 2,718 & 438 \\
\hline $\mathrm{CC}$ coherent $1 \pi^{-}$ & 4,487 & 0 \\
\hline $\mathrm{CC}$ coherent $1 \pi^{+}$ & 0 & 748 \\
\hline other (multi- $\pi$, DIS) & 2,589 & 2,156 \\
\hline total & 71,547 & 30,539 \\
\hline
\end{tabular}

antineutrino mode data, allowing more precise antineutrino cross section measurements. The wrong-sign content is constrained by three measurements: muon angular distributions in quasielastic (CC QE) events, muon lifetimes, and the measured rate of $\mathrm{CC}$ single pion $\left(\mathrm{CC} 1 \pi^{+}\right)$ events [3]. 
Table 2

Wrong-sign extraction uncertainties as obtained from various independent sources in the $\bar{\nu}$ data. The resultant systematic uncertainty on $\bar{\nu}$ cross section measurements is obtained by assuming that wrong-signs comprise $30 \%$ of the total events.

\begin{tabular}{ccc} 
Measurement & $\begin{array}{c}\text { WS } \\
\text { uncertainty }\end{array}$ & $\begin{array}{c}\text { resultant } \\
\text { error on } \sigma_{\bar{\nu}}\end{array}$ \\
\hline $\mathrm{CC} \mathrm{QE} \cos \theta_{\mu}$ & $7 \%$ & $2 \%$ \\
$\mathrm{CC} 1 \pi^{+}$cuts & $15 \%$ & $5 \%$ \\
muon lifetimes & $30 \%$ & $9 \%$ \\
\hline
\end{tabular}

\subsection{Muon Angular Distributions}

The most powerful wrong-sign constraint comes from the observed direction of outgoing muons in CC QE interactions. Neutrino and antineutrino events exhibit distinct muon angular distributions. Due to the antineutrino helicity, the final state muons in $\bar{\nu}_{\mu} \mathrm{QE}$ events are more forward peaked than muons from $\nu_{\mu}$ interactions.

MiniBooNE's angular resolution allows exploitation of this difference by fitting the angular distributions to extract the wrong-sign contribution. Analysis of Monte Carlo data sets determined the accuracy with which the wrong-sign content can be measured using this technique to be $5 \%$ of itself [5]. Including systematic uncertainties and (non-QE) backgrounds increases the uncertainty only to $7 \%$.

\subsection{Muon Lifetimes}

A second constraint results from measuring the rate at which muons decay in the MiniBooNE detector. Due to an $8 \% \mu^{-}$capture probability in mineral oil, positively and negatively charged muons exhibit different effective lifetimes $(\tau=$ $2.026 \mu \mathrm{s}$ for $\mu^{-}$[6] and $\tau=2.197 \mu \mathrm{s}$ for $\mu^{+}$[7]). For CCQE events, we find that the wrong-sign contribution can be extracted with a $30 \%$ statistical uncertainty based solely on this lifetime difference and negligible systematic uncertainties. While not as precise as fits to the muon angular distributions, this particular constraint is unique, as it is independent of kinematics.

\subsection{Single Pion Event Sample}

Our third wrong-sign constraint employs the the fact that antineutrinos do not create any $\mathrm{CC} 1 \pi^{+}$events in the detector-these all stem from neutrinos (Table 1). MiniBooNE identifies $\mathrm{CC} 1 \pi^{+}$events by tagging the two decay electrons that follow the primary neutrino interaction, one from the $\mu^{-}$and one from the $\pi^{+}$decay $[8$. However, $\mathrm{CC} 1 \pi^{-}$events do not pass this requirement because most of the emitted $\pi^{-}$'s are absorbed in carbon, leaving no decay electrons. Applying these cuts to the full sample, which is $70 \%$ antineutrino (RS) interactions, yields an $85 \%$ pure sample of WS neutrino events.

Assuming conservative uncertainties for the antineutrino background events and the $\mathrm{CC} 1 \pi^{+}$ cross section, which is currently being measured by MiniBooNE, we expect a $15 \%$ uncertainty on the wrong-sign content in the beam given $2 \times 10^{20}$ POT. This constraint is complementary to the muon angular distributions because $\mathrm{CC} 1 \pi^{+}$events stem mainly from resonance decays, thus constraining the wrong-sign content at larger neutrino energies.

\subsection{Summary of Wrong Sign Constraints}

0 The three separate techniques to measure the wrong-sign content in the antineutrino data will lend confidence to the antineutrino cross section measurements and greatly reduce their associated systematics. Combined, these three independent measurements (each of which have different systematics) offer a very powerful constraint on the neutrino backgrounds in antineutrino mode (Table 21).

\section{CC Quasi-Elastic Scattering}

MiniBooNE expects more than 40,000 QE interactions in antineutrino mode with $2 \times 10^{20}$ POT before cuts. Using the same QE event selection criteria as the previously reported MiniBooNE neutrino analysis [9] yields a sample of $\sim 19,000$ events, with $75 \%\left(\nu_{\mu}+\bar{\nu}_{\mu}\right)$ QE purity.

Assuming the wrong-sign constraint from Section 2 and conservative errors on the $\nu$ flux, the backgrounds, and event detection, we expect a MiniBooNE measurement of the antineutrino QE 
cross section to better than $20 \%$ with $2 \times 10^{20}$ POT.

\section{NC Single Pion Production}

There has been only one published measurement of the absolute rate of $\bar{\nu}_{\mu} \mathrm{NC} \pi^{0}$ production, with $25 \%$ uncertainty at $2 \mathrm{GeV}$ 10]. This channel is the largest background to future $\bar{\nu}_{\mu} \rightarrow \bar{\nu}_{e}$ oscillation searches.

Applying MiniBooNE's $\nu_{\mu} \mathrm{NC} \pi^{0}$ cuts [1], with no modifications, leaves a sample of antineutrino NC $\pi^{0}$ events with a similar event purity and efficiency. After this selection, we expect 1,650 $\bar{\nu}_{\mu}$ resonant $\mathrm{NC} \pi^{0}$ events and $1,640 \bar{\nu}_{\mu}$ coherent $\mathrm{NC}$ $\pi^{0}$ events assuming $2 \times 10^{20} \mathrm{POT}$ 412]. The WS background of $\sim 1000 \mathrm{WS}$ events will be known from the constraints on the wrong-sign content in the beam as described in Section 2 and the measurement of the $\nu_{\mu} \mathrm{NC} \pi^{0}$ cross section from MiniBooNE neutrino data.

\section{CC Single Pion $\left(\mathrm{CC} 1 \pi^{-}\right)$Production}

MiniBooNE expects roughly 7,000 resonant CC $1 \pi^{-}$with $2 \times 10^{20}$ POT before cuts. As discussed above, most of the emitted $\pi^{-}$'s will be absorbed by carbon nuclei, and will therefore not be selected by the $\mathrm{CC} 1 \pi^{+}$cuts. Nevertheless, these events still have a signature: two Cherenkov rings (one each from the $\mu^{+}$and $\pi^{-}$) and one Michel electron in the vicinity of the $\mu^{-}$. The selection efficiency and purity of such events is unknown at this time. Further investigation is currently underway.

\section{Conclusions}

We have developed three techniques for determining the wrong-sign background in antineutrino mode. The resulting systematic error on any $\bar{\nu}$ cross section measurement due to the wrong sign contamination is around $2 \%$, with a total uncertainty around $20 \%$, which is remarkable for a detector which does not possess event-by-event sign selection. Given this redundant approach, the wrong-sign contamination should not be considered prohibitive to producing meaningful antineutrino cross section [3] and oscillation mea- surements 31314 at MiniBooNE. These techniques may also be useful for other experiments without magnetized detectors which have plans to study antineutrino interactions (e.g. T2K, $\mathrm{NO} \nu \mathrm{A}$, Super-K).

\section{Acknowledgments}

The author is pleased to acknowledge the collaborative efforts of J.M. Link, H.A. Tanka, and G.P. Zeller in developing the ideas in this work.

The MiniBooNE collaboration gratefully acknowledges support from various grants and contracts from the Department of Energy and the National Science Foundation. The author was supported by grant number DE-FG02-91ER0617 from the Department of Energy.

\section{REFERENCES}

1. G. P. Zeller, NuInt02, hep-ex/0312061

2. I. Stancu, these proceedings.

3. MiniBooNE Collaboration, "Addendum to the MiniBooNE Run Plan: MiniBooNE Physics in 2006," available from http://www-boone.fnal.gov/publicpages/loi.ps.gz

4. D. Casper, Nucl. Phys. Proc. Suppl. 112, 161 (2002), hep-ph/0208030

5. H.A. Tanaka, "Estimating Wrong Sign Contamination in Negative Polarity Horn Data", BooNE Memo (2004)

6. T.Suzuki et al., Phys. Rev. C35, 2122 (1987).

7. S.Eidelman et al., Phys. Lett. B592, 33 (2004).

8. M. O. Wascko, DPF04, hep-ex/0412008

9. J. Monroe, Moriond 2004, hep-ex/0406048.

10. H.Faissner, et al., Phys. Lett. 126B, 230 (1983).

11. J. L. Raaf, NuInt04, hep-ex/0408015.

12. D. Rein and L. M. Sehgal, Nucl. Phys. B223, 29 (1983).

13. J. Monroe, " $\nu_{\mu}$ Disappearance Studies for the Fall 2004 Letter of Intent", BooNE Memo (2004)

14. A.A. Aguilar Arevalo, "MiniBooNE Oscillation Sensitivity in Antineutrino Running Mode", BooNE Memo (2004) 